\title{
Energy Efficient CO-GPS with Reduced Duty Cycle Environment Monitoring
}

\author{
P.D. Karale ${ }^{1}$, S.B. Shinde ${ }^{2}$ \\ Dept of Electronics and Telecommunication, JSPM Technical Campus, Savitribai Phule Pune University, Pune, India ${ }^{1,2}$
}

\begin{abstract}
Advancements in silicon technology, embedded systems, sensors, micro electro mechanical systems and wireless communications have led to the emergence of embedded wireless sensor networks (EWSNs). System explained here is an embedded system which uses ARM \& a wireless sensor network. Typical widely available GPS receivers consume high energy, which limits its usefulness in any applications. In many sensing scenarios, preprocessing of location information is adequate at the time of data uploading to a server, Cloud-Offloaded GPS (COGPS) solution is designed that allows a sensing device to forces to manage duty-cycle its GPS receiver and log just raw data GPS signal for post processing. Cloud can compute good quality GPS location can be derived by using publicly available information such as GNSS satellite ephemeris and an Earth elevation database service, from a few milliseconds of raw data. GPS Module give us the output in terms of Latitude \& Longitude of the location. Using GPRS module at we can collect all the information from the wireless sensor nodes \& we can transmit it to the server. By using webpage, all the status of the system can be monitored.
\end{abstract}

Keywords: Embedded wireless sensor networks, Cloud-offloaded GPS (CO-GPS), Coarse time navigation (CTN), Webpage, Server, GPS receiver.

\section{INTRODUCTION}

Location determination is a fundamental service in mobility. In outdoor applications such as wildlife tracking [15], participatory environmental sensing [16] and personal health and wellness applications, GPS is the most common location sensor. GPS receiving, although becoming increasingly more common and lower in cost, is processingintensive and energy consuming.

In this system, the problem of energy consumption in GPS receiving is addressed, by splitting the GPS location sensing into a device part and a cloud part. Many mobile sensing applications are delay-tolerant. Instead of determining the location at instant time, each data sample is collected and we can compute the locations off-line after the data is uploaded to a server. This is quite different from the turn-by-turn navigation scenario that most standalone GPS devices are designed for. The benefit is even more significant if the data uploading energy is amortized over many data samples much of the information necessary to compute the location of a GPS receiver is available on line. The only information that the device must provide is a rough notion of time, the set of visible satellites, and the "code phase" information from each visible satellite. If location is derived without decoding any data from the satellites, there is significant opportunity to duty-cycle the receiver.

\section{RELATED WORK}

Efficient power saving techniques must be designed and developed to control energy consumption by sensor nodes, which are tiny and can easily get damaged if battery power fails.

Global Positioning System (GPS) is main technique used in Location-Based Service (LBS) like wild life tracking, smartphones, cars etc. However, due to heavy computations, GPS increases the energy consumption. Dohee Kim, Soyoon Lee, and Hyokyung Bahn [1], proposed EEPS (Energy-Efficient Positioning Scheme) to overcome this problem. Concept of interesting regions of user is introduced here. Interesting region is the term used for frequently visited locations of user. Based on accuracy requirement, interesting region and battery level, EEPS adaptively performs the positioning computation of each application. By considering simulations results of various real applications and scenarios this paper claims that EEPS reduces $50.1 \%$ of energy consumption compared to traditional GPS. Also, satisfies the accuracy requirement of each application.

Though GPS provides high accuracy in environmental monitoring applications, high energy consumption is main setback of GPS sensing. GPS sampling rate frequency i.e. duty cycle strongly emphasizes energy consumption of sensor nodes. Thus reducing battery life as well as network lifetime. [2]Xiaohan Li, Fengpeng Yuan and JanneLindqvist have proposed to guarantee accuracy of location determination by formulating most energy efficient duty cycle of GPS. Results of real life traffic monitoring results illustrated in this paper states to save $78 \%$ energy,when 
sampling period is increased beyond 120 seconds. Loss of accuracy stabilize around 23\%, compared to continuous sampled GPS.

[3]Kaikai Liu, Qiuyuan Huang, Jiecong Wang, Xiaolin Li and Dapeng Oliver Wu proposed a socialaidedcooperative location optimization (Coloc) scheme. The proposed scheme discusses about smartphone nodes, yet same can be reviewed in environmental monitoring perspective. Cooperative location optimization scheme fuses GPS positions of multiple collocated smartphones in a social network, or by neighborhoodbasedweighted least-squares estimation when relative distancesbetween smartphones are available. This improves positioning accuracy and also reduces energy consumption of system. Co-located smartphone nodes shares location information and lower update rate of the GPS module without compromising the accuracy. Experiments conducted stationary and moving scenarios validates that, cooperativelocalization schemecan perform satisfactorily.

An adaptive data compression technique is proposed by Beihua Ying, in order to improve the energy efficiency for the whole network. This paper [4], proposes realization of a real-time adjustment of compression strategy. Algorithm for adaptive data compression technique provides optimal execution strategies for each node in wireless sensor networks. In this algorithm prediction and feature extraction of several relevant parameters is considered. The simulation results prove that; the proposed compression scheme completes data communication for all nodes in a network with minimal energy consumptions. The maximum deviation against the ideal condition is less than $5 \%$.

Based on the IEEE 1451 standard, NihalKularatna and B.H.Sudantha [5] proposed environmental air pollution monitoring system. This paper explains design and implementation of STIM (smart transducer interface module). Like typical wireless sensor networks, STIM is combination of microcontroller and group of various sensors like $\mathrm{CO} 2, \mathrm{CO}$, NO2, and O3. Graphical representation of results is presented using. STIM and the PC are connected via IEEE 1451 standard transducer independent interface.

[6] Design and development of a ZigBee based energy efficient environmental monitoring, alerting and controlling system for environmental monitoring is proposed in this paper. K.Lokesh Krishna, J.Madhuri and Dr.K.Anuradha illuminates about wireless monitoring of agricultural field will allow the farmers to lessen the human power and helps to keep real time accurate data of changing parameters in the field. The proposed system helps to keep track of various environmental parameters such as temperature, humidity, gas density, soil humidity and light availability. Being highly robust, proposed system is highly recommended for environment monitoring over large areas.

In "Wireless sensor networks: a survey" [7], I.F. Akyildiz, W. Su, Y. Sankarasubramaniam, E. Cayirci reviews multiple available and under research wireless sensor network methodology. The concept of sensor network is explained with the reference of microelectro-mechanical system, electronics and wireless communication techniques. Authors treat sensing task and network application as separate entity and review is provided sating sensor networks must overcome constraints as fault tolerance, scalability, expenses and efficient energy consumption. Need for application based wireless ad hoc networking techniques is stated here.

IOT is trending factor in environmental monitoring. Remote data monitoring is mandatory because human presence is limited due to many factors such as unfriendly geographical conditions, temperature, toxic gases. [8] P.Suchetha and T. VenuMadhav proposed a new method for a Wireless Sensor Node to become an Internet of Thing Sensor. Computed data is communicated using standard IEEE 802.15.4 and uploaded to internet. Real time monitoring and accuracy are key parameters of this research.

In [9], L-SYNC synchronisation protocol design is illustrated for Wireless Sensor Networks. This protocol provides efficiency for homogeneous network topology clustering. Reduction of number of hops between clusters is strength of the proposed design. L-sync clustering according to larger degree technique reduces cluster overlapping. For analysing results the synchronization precision of two remote nodes is considered. L-SYNC does have limitations for heterogeneous networks. For heterogeneous network topologies, L-SYNCng (L-SYNC next generation) protocol is proposed here. For better synchronization and scalability, L-SYNCng protocol is good but it is unreliable in noisy environments.

\section{METHODOLOGY}

Relevant details should be given including experimental design and the technique (s) used along with appropriate statistical methods used clearly along with the year of experimentation (field and laboratory). Fundamental aim of the system is to establish Energy Efficient wireless sensor network between client and server using energy efficient GPS sensing with cloud offloading technology. The system contains two parts. One is transmitter node and another one is receiver part. The transmitter part consists of sensors, microcontroller and GPS, and the receiver part consist of a PC interfaced with GPRS. System monitors environmental factors as $\mathrm{CO} 2$ concentration, temperature, humidity and light intensity.Also, system get the current position (longitude, latitude and elevation) and timing (GMT) information through Global Positioning System (GPS). Sensed data and location information is displayed on LCD. When sensor parameters cross threshold limit, controller sends data to server with the help of GPRS module. This sensor data stored in server is accessed from anywhere in the world with help of Webserver page on PC using TCP/IP protocol. 


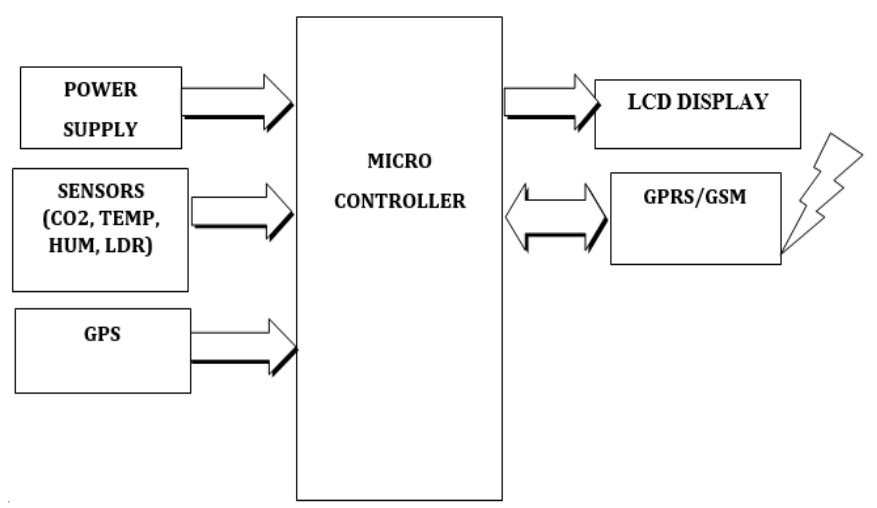

Figure 1: System Architecture

GPS receivers are energy hungry devices. Reasons behind high energy need are -

- Ephemeris i.e. orbital information of satellite is received at low data rate. To receive full data packet, GPS receiver remains on for long time (up to 30 seconds).

- Due to high speed of satellite, previous collected information is not valid. So GPS chip takes more time to calculate location.

- Location calculations need powerful CPU

Implemented system simply turns off GPS receiver instead of turning off entire GPS chip. This compensate in time required for location information. Location computation is separated between cloud and local processors. Received GPS information is processed off-line using high speed processor. This method is called as Cloud-Offloaded GPS(COGPS).

Energy efficient cloud offloaded technology is developed on the basis of coarse time navigation (CTN) technique. CTN technique acquires location data like typical GPS receivers by using 1023-bit GPS Gold codes. 1023-bit GPS golden codes are 1023 bit long Coarse/Acquisition codes, one for each satellite. CTN is ideal for design base of energy efficient CO-GPS design is due to the advantage of satellite ephemeris data published by GPS monitoring organizations like NASA. Ephemeris is orbital information of satellite that is sending data.

Ephemeris data is very detailed and valid for four hours. When receiver locates satellite, it obtains ephemeris directly from the satellite. This gives advantage in energy saving aim. CTN is used to speed up first location lock of satellite. Location lock of satellite have further advantage in maintaining real time clock with satellite synchronization and also, lessen dependency of reference location. GPS receiver node of system need to be time synchronized with satellite only once.

Thus effectively CO-GPS receiver have very short duty cycle for long time running monitoring solutions. For embedded application sensors in environment monitoring cellular connections are not realistic. Hence CTN principle of removing reference location dependency serves the purpose. When system requires to locate itself, it simply turns on GPS receiving front end and records a few milliseconds of GPS signal. Processor derive the receiver node location from few millisecond data and coarse time stamp.

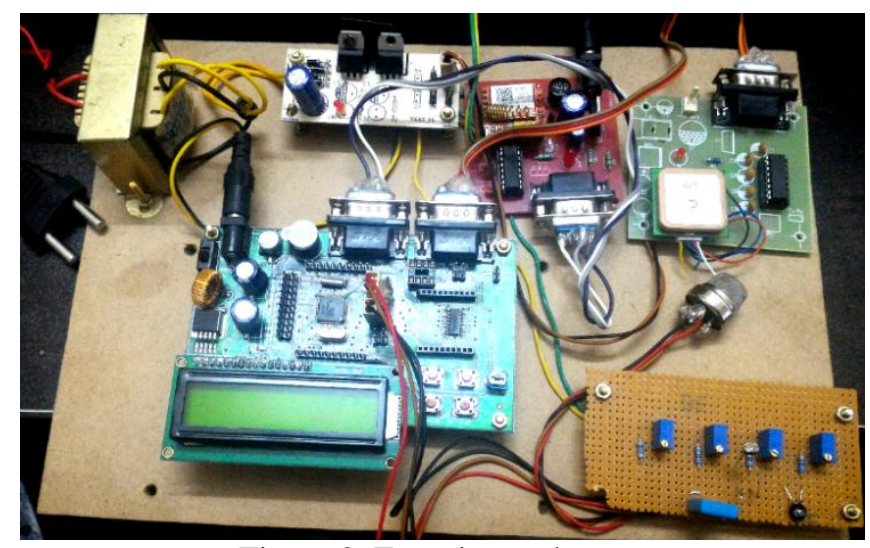

Figure 2: Experimental setup 
ARM LPC2148 controller is used to build sensor node. NXP's development board is ideal for fast embedded communication application. The board provides extra strength to the connector joints for increased reliability. Temperature sensor is used to sense environment temperature. Humidity sensor (HUM) is a device that measures the relative humidity of in a given area. The LDR is used to measure the light intensity. Gas sensor detects concentration of $\mathrm{CO} 2$ of environment.

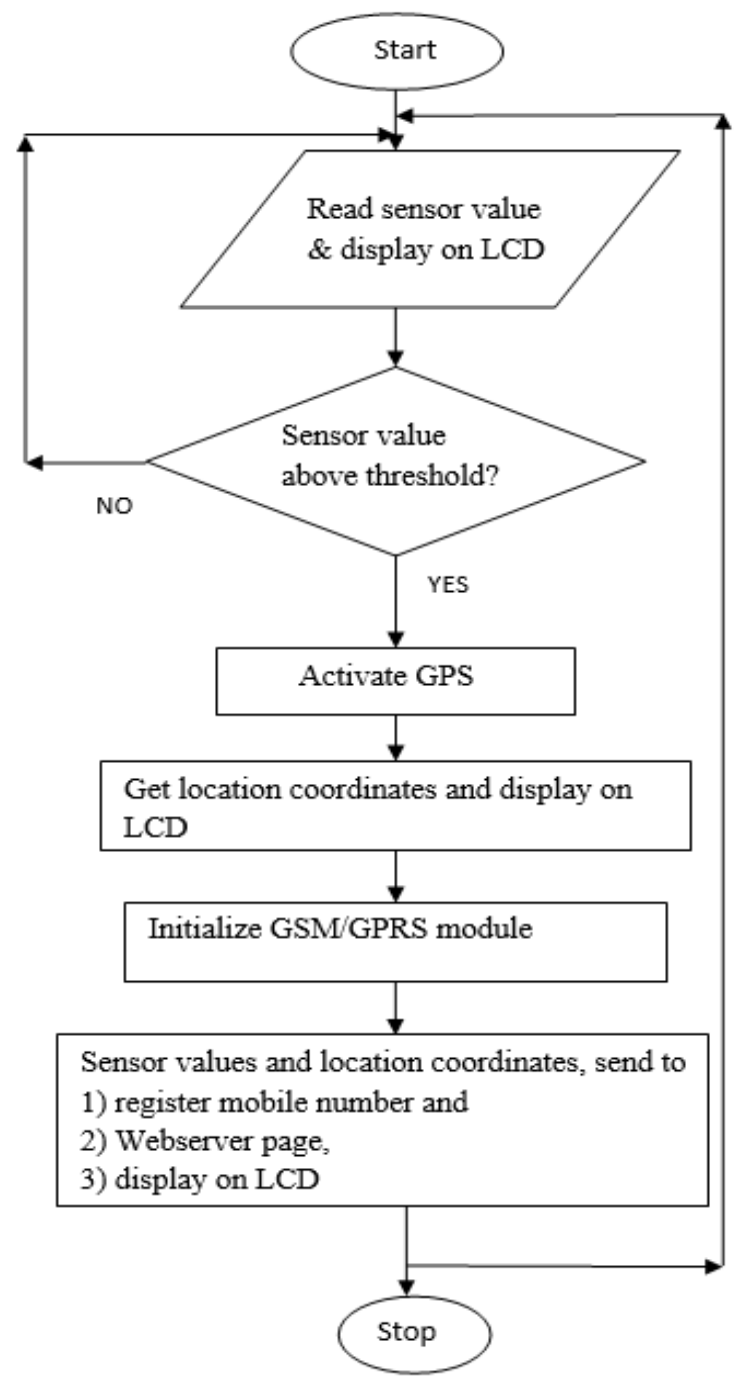

Figure 3: Flowchart of the system

GPS receiver front end is build using G65 chip and controller LPC2148. A GPS modem is used to get the signals and receive the signals from the satellites. In the system, GPS modem get the signals from the satellites and those are given to the microcontroller. The signals are in the form of the coordinates; these are represented in form of the latitudes, longitudes and altitudes. GPRS section consists of a GPRS modem. The modem communicates with microcontroller using serial communication. The modem is interfaced to microcontroller using MAX 232, a serial driver. The Global Packet Radio Service is a TDMA based digital wireless network technology that is used for connecting directly to internet. GPRS module will help us to post data in the web page directly.GSM modem section consists of a GSM modem. The modem communicates with microcontroller using serial communication. The modem is also interfaced to microcontroller using MAX 232, a serial driver. SIM card is used to identify the user's account, in GSM phones.

\section{RESULTS AND DISCUSSION}

Webpage Server GUI welcome message: When webpage is opened on "GPRS enabled PC", welcome message is displayed. 
Vol. 6, Issue 6, June 2017

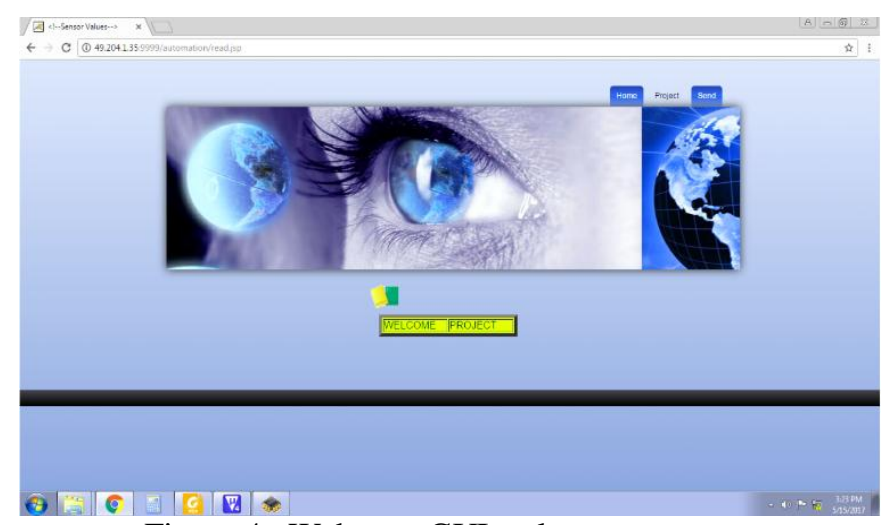

Figure 4 : Webpage GUI welcome message

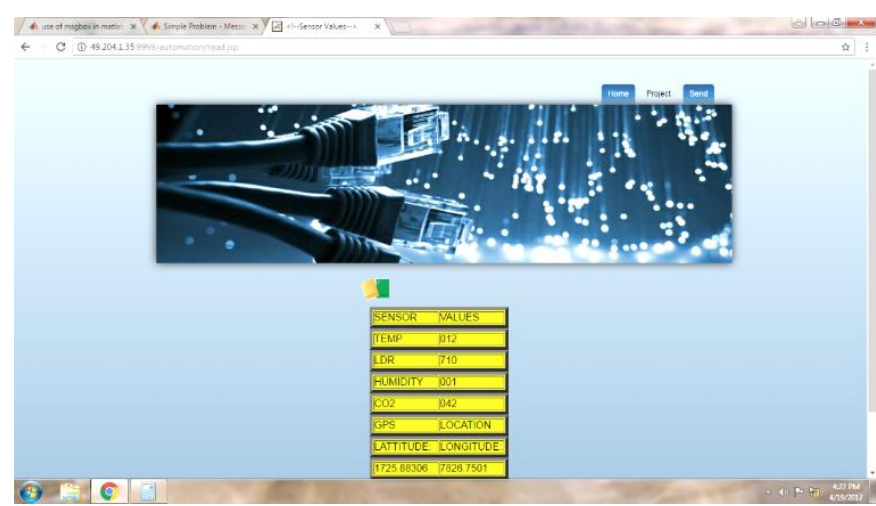

Figure 5 : Sensor data and location information on webpage

System continuously monitors sensor data and when sensor data crosses threshold limits, data is uploaded to webpage. Latitude and longitude of sensor node are also uploaded.

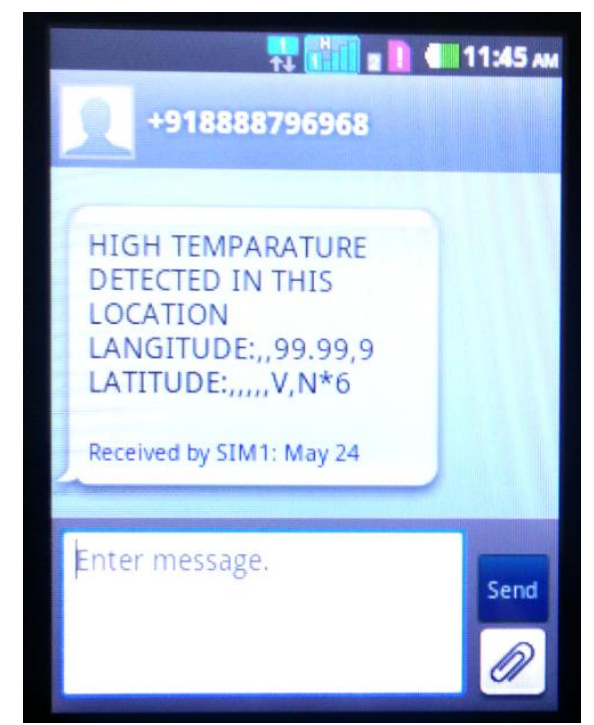

Figure 6: Sensor data and location information on GSM phone

When sensor data crosses threshold limits, data and location information SMS is also sent to GSM phone. Sensor data can be continuously monitored on LCD display also.

\section{CONCLUSION AND FUTURE SCOPE}

An architecture for energy efficient GPS \& embedded wireless sensor networks (EWSNs) is proposed here. The increased energy efficient GPS power offered by embedded sensor nodes prove useful for information fusion, 
encryption, network coding. The energy efficient CO-GPS technique for environmental monitoring will benefit in terms of prolonged battery life and hence, increases life span of embedded sensor network.

\section{REFERENCES}

[1] Dohee Kim, Soyoon Lee, and HyokyungBahn, Dept. of Computer Science and Engineering EwhaWomans University Seoul, Republic of Korea "An Energy-Efficient Positioning Scheme for Location-Based Services in a Smartphone" in 2016 IEEE 22nd International Conference on Embedded and Real-Time Computing Systems and Applications, pp 139-148

[2] Xiaohan Li, Fengpeng Yuan and JanneLindqvist Rutgers University, "Feasibility of Software-Based Duty Cycling of GPS for Trajectory-Based Services", in 2016 13th IEEE Annual Consumer Communications \& Networking Conference (CCNC) on 9-12 Jan. 2016, pp. 293-306

[3] Qiuyuan Huang, Jiecong Wang, Xiaolin Li, Dapeng Oliver and Kaikai Liu "Improving GPS Service via Social Collaboration" in 2013 IEEE 10th International Conference on Mobile Ad-Hoc and Sensor Systems, 2013, pp. 394-401

[4] Beihua Ying, School of Information Science and Engineering, Ningbo Institute of Technology, Zhejiang University, Ningbo, "An Adaptive Compression Algorithm for Energy-Efficient Wireless Sensor Networks" in Advanced Communication Technology (ICACT), 2017 19th International Conference Bongpyeong, South Korea on 19-22 Feb. 2017, pp. 861-868

[5] N. Kularatna and B. H. Sudantha, "An environmental air pollution monitoring system based on the IEEE 1451 standard for low cost requirements," Apr. 2008, pp. 415-422

[6] K.Lokesh Krishna, J.Madhuri, Dr.K.Anuradha, "A ZigBee based Energy Efficient Environmental Monitoring Alerting and Controlling System", International Conference On Information Communication And Embedded Systems IEEE, on 25-26 Feb 2016, pp. 978-1-5090-2552$7 / 16 /$

[7] I.F. Akyildiz, W. Su, Y. Sankarasubramaniam, E. Cayirci, "Wireless sensor networks: a survey" in IEEE Wireless Communications Volume:14, Issue:6, December 2007, pp. 32 - 39

[8] P. Suchetha, T. Venu Madhav, "Energy Efficient Environment Monitoring System Based on the IEEE 802.15.4 Standard for Low Cost Requirements" in IOSR Journal of Electronics and Communication Engineering (IOSR-JECE) e-ISSN: 2278-2834, p- ISSN: 2278-8735. PP $01-08$

[9] Alireza Shameli Sendi, Naser Ezzati Jivan, Masoume Jabbarifar, Alireza Sadighian in "A Reliable and Efficient Time Synchronization Protocol for Heterogeneous Wireless Sensor Network" revised November 1, 2010; accepted November 8, 2010 wireless Sensor Network, 2010, 2, pp. 910-918

[10] A. Arora, P. Dutta, S. Bapat, V. Kulathumani, H. Zhang, V. Naik, V. Mittal, H. Cao, M. Demirbas, M. Gouda, Y. Choi, T. Herman, S. Kulkarni, U. Arumugam, M. Nesterenko, A. Vora, "A line in the sand: A wireless sensor network for target detection, classification, and tracking," Comput. Netw., vol. 46, no. 5, pp. 605-634, Dec. 2004.

[11] Abdul Kareem Shaik, Ashok Kumar, Dr. MadharSaheb Shaik Dept of ECE CMR Engineering College Hyderabad, Telangana, "Implementation of Wireless Sensor Networks for Industrial Applications Using The Multi-Core Architecture" in International Journal of Engineering Research and Applications on Developments, Advances \& Trends in Engineering Sciences (NCDATES- 09th \& 10th January 2015)

[12] K. Chintalapudi, A. Padmanabha Iyer, and V. N. Padmanabhan,"Indoor localization without the pain," in Proc. 16th Annu. Int.Conf. Mobile Comput. Netw., Chicago, IL, USA, Sep. 20-14, 2010,pp. 173-184.

[13] M. Horemuz and J. V. Andersson, "Polynomial interpolation of GPS satellite coordinates," GPS Solut, vol. 10, pp. 67-72, 2006.

[14] X. Jiang, C.-J. M. Liang, F. Zhao, K. Chen, J. Hsu, B. Zhang, and J. Liu, "Demo: Creating interactive virtual zones in physical spacewith magnetic-induction," in Proc. 9th ACM Conf. Embedded Netw.Sensor Syst., Seattle, WA, USA, Nov. 4-7, 2011, pp. 431-432.

[15] A. Liu, M. Faulkner, M. Olson and K. M. Chandy, "Rapid detection of rare geospatial events: Earthquake warning applications, in Proc. 5th ACM Int. Conf. Distrib. Event-Based Syst.,NewYork,NY, USA, Jul. 11-15 2011, pp. 89-100.

[16] H. S. Ramos, A. Kansal, T. Zhang, J. Liu and N. B. Priyantha "LEAP: A low energy assisted GPS for trajectory-based services,"in Proc. 13th Int. Conf. Ubiquitous Comput., Beijing,China, Sep. 17-21, 2011, pp. 335-34.

[17] E. D. Kaplan and C. J. Hegarty,Understanding GPS: Principles and Applications, 2nd ed. Norwood, MA, USA: Artech House, 2005.

\section{BIOGRAPHIES}

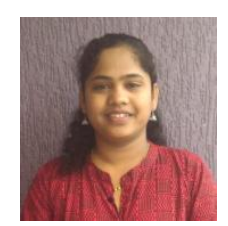

Priya Karale is pursuing ME in Embedded \& VLSI in E\&TC department of JSPM, Narhe Technical Campus, Pune. She has completed B.E. from SRTMU University, Nanded. Her research interest is wireless communication technology.

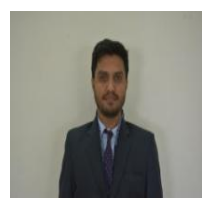

Mr. Sagar Shinde is working as a Assistant Professor in E\&TC department of JSPM, Narhe Technical Campus, Pune. He has completed B. E from DYPIET, Pune and M.E from NMU, Jalgaon. He has total 8 years of experience in teaching, published two patents and no. Of papers in peer reviewed journals and editorial board member in 3 international journals. 